\title{
EDITORIAL
}

\section{Insights in Plant-Microbe Interaction Through Genomics Approach (Part II)}

\author{
Jagajjit Sahu ${ }^{1}$, Anukool Vaishnav ${ }^{2}$ and Harikesh B. Singh ${ }^{3, *}$
}

\author{
${ }^{I}$ National Center for Cell Sciences (NCCS), University of Pune Campus, University Road, Ganeshkhind, Pune-411007, \\ Maharashtra, India; ${ }^{2}$ Department of Biotechnology, Institute of Applied Sciences and Humanities, GLA University, \\ Mathura-281121, Uttar Pradesh, India; ${ }^{3}$ Somvanshi Research Foundation, 13/21 Vikas Nagar, Lucknow-226022, Uttar \\ Pradesh, India
}

In continuation with part I, this part describes the use of genetically modified microbes, rhizospheric microbial communities, various molecular techniques and omics approaches to shed the light on understanding plant-microbe interactions [1]. Due to the long ongoing controversies regarding the use of application of genetically engineered microbial inoculants in the field, it has been a hot topic since the last two decades. The authors have been able to provide descriptive information regarding the same with special application towards plant-microbe interactions. A bright study carried out with the help of omics technologies, especially bioinformatics approaches to understand the microbial communities in coal samples.

Sudheer and co-authors presented a mini-review on engineered microbes and their applications in sustainable agriculture [2]. Authors emphasized especially on methods to shape the rhizosphere zone, which can ultimately promote the beneficial microbial population in the soil. Microbial communities in the soil can be diverse and a deeper understanding of their nature can help plants to provide enhanced stress tolerance, disease resistance, nutrient acquisition and also many other important factors. There are various efficient genetic engineering/molecular techniques which are available and being used to attain better plant health and yield. The authors provided a simpler version of understanding plant-microbe interaction and how the networks work in the soil. Also mentioned briefly about the microbial inoculants and their function as biofertilizers, biocontrol agents, and in biotic and abiotic stress tolerance. Though the application of genetically engineered microbial inoculants in the field is still not approved fully, the potential of these cannot be denied. Upon more research and better as well as safe practices, these can be used as huge assets to achieve sustainable agriculture.

In addition, a review is presented on genomics approaches to provide insights into the role of rhizospheric microbial communities in plant-microbe synergy for enhanced phytoremediation [3]. This review aims at drawing an understanding of genetic approach integrated with bioremediation of contaminated sites. Authors have described extensively on phytoremediation and the role of predominant rhizospheric microbial communities in it. In the later part, the application of genetically modified plants and microbes for phytoremediation has been described. The cutting-edge genomic approaches such as CRISPR (clustered regularly interspaced short palindromic repeats) technology was illustrated in plant-microbe synergy. Authors also presented a table containing various plants, microbes, target pollutants and their enhanced effect on phytoremediation.

Another review by Sharma and co-authors provided insights into overall omics technologies for plant-microbe interaction [4]. The review tries to bring together important aspects such as plant growth promotion, nitrogen fixation, stress suppressions and metabolic interactions along with the recent studies on various advanced omics approaches. The basics of various types of plant-microbe interactions have been stated briefly with a vivid description of various molecular techniques. It continues with an extensive discussion of applications of next generation sequencing techniques and meta-omics approaches in unravelling plant-microbe interaction understandings. The authors also discussed the research gaps and future prospects of omics approaches with respect to plant-microbe interaction studies.

Jha and team carried out bioinformatics analyses of the metagenomic data obtained from the West Jharia coal seam, Moonidih, Jharkhand [5]. Pyrosequencing was performed on DNA extracted from the coal samples following by bioinformatics analyses which are taxonomic assignment and functional analysis. The upshot of the study helped in revealing important information regarding biodiversity such as the dominance of hyperthermophilic archaea Pyrococcus furiosus along with Thermococcus kodakarensis present in the underground coal mines of Jharia. The results also revealed the presence of dissimilatory sulfite reductase and formylmethanofuran dehydrogenase enzymes involved in sulfite reduction and methanogenesis processes respectively. Much information about real efficiency of coal degrading microbes is still hidden. The study has been able to provide preliminary as well as extensive insight into meta-genomic information such as taxonomic and functional diversity of the microbial communities in coal mines.

*Address correspondence to this author at the Somvanshi Research Foundation, 13/21 Vikas Nagar, Lucknow-226022, Uttar Pradesh, India; Tel: +915426702583; +91542-6702576; Cell: 09415355571; E-mail: hbsinghbhu01@gmail.com 


\section{AUTHORS' CONTRIBUTIONS}

All authors JS, AV, HBS, contributed in writing this Editorial article for the research topic "Insights in plant-microbe interaction through genomics approach (Part-II)".

\section{REFERENCES}

[1] Sahu, J.; Vaishnav, A.; Singh, H.B. Insights in plant-microbe interaction through genomics approach (Part I). Curr. Genomics, 2020, 21(3), 155-156. https://doi.org/10.2174/138920292103200625161718

[2] Sudheer, S.; Bai, R.G.; Usmani, Z.; Sharma, M. Insights on engineered microbes in sustainable agriculture: biotechnological developments and future prospects. Curr. Genomics, 2020, 21(5), 321-333.

https://doi.org/10.2174/1389202921999200603165934

[3] Agarwal, P.; Giri, B.S.; Rani, R. Unravelling the role of rhizospheric plant-microbe synergy in phytoremediation: a genomic perspective. Curr. Genomics, 2020, 21(5), 334-342.

https://doi.org/10.2174/1389202921999200623133240

[4] Sharma, M.; Sudheer, S.; Usmani, Z.; Rani, R.; Gupta, P. Deciphering the omics of plant-microbe interaction: perspectives and new insights. Curr. Genomics, 2020, 21(5), 343-362.

https://doi.org/10.2174/1389202921999200515140420

[5] Jha, P.; Singh, J.; Vidyarthi, A.S.; Prasad, R. Unveiling the biodiversity of hyperthermophilic archaea in jharia coal mines: potential threat to methanogenesis? Curr. Genomics, 2020, 21(5), 363-371.

https://doi.org/10.2174/1389202921999200605151722 